Proyecciones

Vol. 26, No 1, pp. 27-35, May 2007.

Universidad Católica del Norte

Antofagasta - Chile

DOI: 10.4067/S0716-09172007000100002

\title{
UNIFORM CONVERGENCE OF MULTIPLIER CONVERGENT SERIES
}

\author{
CHARLES SWARTZ \\ NEW MEXICO STATE UNIVERSITY
}

Received: December 2006. Accepted: February 2007

\begin{abstract}
If $\lambda$ is a sequence $K$-space and $\sum x_{j}$ is a series in a topological vector space $X$, the series is said to be $\lambda$-multiplier convergent if the series $\sum_{j=1}^{\infty} t_{j} x_{j}$ converges in $X$ for every $t=\left\{t_{j}\right\} \in \lambda$. We show that if $\lambda$ satisfies a gliding hump condition, called the signed strong gliding hump condition, then the series $\sum_{j=1}^{\infty} t_{j} x_{j}$ converge uniformly for $t=$ $\left\{t_{j}\right\}$ belonging to bounded subsets of $\lambda$. A similar uniform convergence result is established for a multiplier convergent series version of the Hahn-Schur Theorem.
\end{abstract}


Let $X$ be a Hausdorff topological vector space and $\sum x_{j}$ a (formal) series in $X$. The series $\sum x_{j}$ is said to be bounded multiplier convergent if the series $\sum_{j=1}^{\infty} t_{j} x_{j}$ converges in $X$ for every $t=\left\{t_{j}\right\} \in l^{\infty}$ ([D]). If $X$ is either a locally convex space or a metric linear space and $\sum x_{j}$ is bounded multiplier convergent, then the series $\sum_{j=1}^{\infty} t_{j} x_{j}$ actually converge uniformly for $t=\left\{t_{j}\right\} \in l^{\infty},\|t\|_{\infty} \leq 1$ ([Sw1], [Sw2]8.2.7). A series $\sum x_{j}$ in $X$ is subseries convergent if the subseries $\sum x_{n_{j}}$ converges in $X$ for every subsequence $\left\{n_{j}\right\}$. If $\sigma \subset \mathbf{N}$, let $C_{\sigma}$ denote the characteristic function of $\sigma$ and if $x$ is any sequence, let $C_{\sigma} x$ denote the coordinatewise product of $C_{\sigma}$ and $x$. Thus, the series $\sum x_{j}$ is subseries convergent iff $\sum_{j=1}^{\infty} C_{\sigma}(j) x_{j}=\sum_{j \in \sigma} x_{j}$ converges for every $\sigma \subset \mathbf{N}$. A similar uniform convergence result holds for subseries convergent series. Namely, if $\sum x_{j}$ is subseries convergent, then the series $\sum_{j=1}^{\infty} C_{\sigma}(j) x_{j}$ converge uniformly for $\sigma \subset \mathbf{N}$ ([Sw2]8.1.2). Another uniform convergence result holds for subseries and bounded multiplier convergent series in the subseries and bounded multiplier convergent versions of the Hahn-Schur Theorem ([Sw1],[Sw2]8.1 and 8.2). We consider conditions under which the same conclusions hold if the multiplier space $l^{\infty}$ is replaced by more general sequence spaces.

Let $\lambda$ be a vector space of scalar sequences which contains $c_{00}$, the space of all sequences which are eventually 0 , and which is equipped with a vector Hausdorff topology under which the coordinate functionals $t=$ $\left\{t_{j}\right\} \rightarrow t_{j}$ are continuous for every $j \in \mathbf{N}$ (i.e., $\lambda$ is a $\mathrm{K}$ space ([B]7.2.2)). Let $\Lambda \subset \lambda$. The series $\sum x_{j}$ in $X$ is $\Lambda$ - multiplier convergent if the series $\sum_{j=1}^{\infty} t_{j} x_{j}$ converges in $X$ for every $t=\left\{t_{j}\right\} \in \Lambda$ ([FP],[Sw2]8.3); thus, a series is $l^{\infty}$-multiplier convergent iff the series is bounded multiplier convergent and a series is subseries convergent iff the series is $m_{0}$ multiplier convergent, where $m_{0}=\operatorname{span}\left\{C_{\sigma}: \sigma \subset \mathbf{N}\right\}$ is the sequence space of all sequences with finite range. It is natural to ask if a uniform convergence result as above for subseries and bounded multiplier convergent series holds for $\lambda$-multiplier convergent series ;i.e., if $\sum x_{j}$ is $\lambda$-multiplier convergent, do the series $\sum_{j=1}^{\infty} t_{j} x_{j}$ converge uniformly for $t=\left\{t_{j}\right\}$ belonging to certain families of bounded subsets of $\lambda$ ? Example 5 shows that such a result does not hold in general, but we show in Theorem 3 that such a result does hold if certain subsets of the multiplier space $\lambda$ satisfy a gliding hump property called the signed strong gliding hump property. We also show that a similar uniform convergence result holds for series in a version of the Hahn-Schur Theorem for $\lambda$-multiplier convergent series when certain subsets of the multiplier space $\lambda$ satisfy the signed strong gliding hump property. 
We begin by defining the gliding hump property which we will employ. An interval in $\mathbf{N}$ is a set of the form $[m, n]=\{k \in \mathbf{N}: m \leq k \leq n\}$ where $m \leq n$; a sequence of intervals $\left\{I_{k}\right\}$ is increasing if $\sup I_{k}<\inf I_{k+1}$. A sign is a variable assuming the values $\{ \pm 1\}$. Let $\Lambda \subset \lambda$. The subset $\Lambda$ has the it signed strong gliding it hump property (signed-SGHP) if for every bounded sequence $\left\{x^{k}\right\} \subset \Lambda$ and for every increasing sequence of intervals $\left\{I_{k}\right\}$, there exists a subsequence $\left\{n_{k}\right\}$ and a sequence of signs $\left\{s_{k}\right\}$ such that $x=$ $\sum_{k=1}^{\infty} s_{k} C_{I_{n_{k}}} x^{n_{k}}$ [coordinate sum] belongs to $\Lambda$. The subset $\Lambda$ has the strong gliding hump property (SGHP) if the signs above can all be chosen to equal to1; the SGHP has been employed on numerous occasions ([N ], [Sw2], [SS]). The idea of multiplying the "humps" $C_{I} x$ by signs was introduced by Stuart ([St1],[St2]) and used to treat weak sequential completeness of $\beta$-duals. For example, the space $l^{\infty}$ has the SGHP and the methods of [BSS] can be used to construct other spaces with SGHP. Let $M_{0}$ be the subset of $m_{0}$ consisting of the sequences of 0's and 1's, $M_{0}=\left\{C_{\sigma}: \sigma \subset \mathbf{N}\right\}$. Then the subset $M_{0}$ has SGHP but the space $m_{0}$ does not have SGHP. We now show that the space of bounded series $b s$ ([B]) has the signed-SGHP but fails SGHP; Stuart showed that bs has the signed weak gliding hump property but not the weak gliding hump property and we essentially just use his proof ([St1],[St2]). Recall that $b s$ is the space of all sequences $t=\left\{t_{j}\right\}$ such that $\|t\|=\sup _{n}\left|\sum_{j=1}^{n} t_{j}\right|<\infty$ equipped with this norm ([B]1.2); an equivalent norm to \|\| is given by $\|t\|^{\prime}=\sup \left\{\left|\sum_{k \in I} t_{k}\right|: I\right.$ an interval $\}$. If $x=\left\{x_{j}\right\}$ and $y=\left\{y_{j}\right\}$ are sequences, we write $x \cdot y=\sum_{j=1}^{\infty} x_{j} y_{j}$ for the formal dot product of $x$ and $y$ when the series converges.

Example 1. bs has signed-SGHP. Actually, $b s$ has an even stronger property than signed-SGHP; it is not necessary to pass to a subsequence in the definition of signed-SGHP. Let $\left\{I_{k}\right\}$ be an increasing sequence of intervals and $\left\{t^{k}\right\} \subset$ bs be bounded. Put $M=\sup \left\{\left|\sum_{j \in I} t_{j}^{k}\right|: k \in \mathbf{N}, I\right.$ an interval in $\mathbf{N}\}<\infty$. Define signs inductively by setting $s_{1}=\operatorname{sign} C_{I_{1}} \cdot t^{1}$ and $s_{n+1}=-\left[\operatorname{sign} \sum_{k=1}^{n} s_{k} C_{I_{k}} \cdot t^{k}\right]\left[\operatorname{sign} C_{I_{n+1}} \cdot t^{n+1}\right]$. Put $y=\sum_{k=1}^{\infty} s_{k} C_{I_{k}} t^{k}$; we show $\|y\| \leq 2 M$. We first show by induction that $\left|\sum_{j=1}^{\max I_{n}} y_{j}\right| \leq M$ for every $n$. For $n=1,\left|\sum_{j=1}^{\max I_{1}} y_{j}\right|=\left|\sum_{j \in I_{1}} s_{1} t_{j}^{1}\right| \leq M$. Suppose the inequality holds for $n$. Then $\left|\sum_{j=1}^{\max I_{n+1}} y_{j}\right|=\left|\sum_{j=1}^{\max I_{n}} y_{j}+\sum_{j \in I_{n+1}} y_{j}\right| \leq$ $M$ since $\left|\sum_{j \in I_{n+1}} y_{j}\right|=\left|\sum_{j \in I_{n+1}} s_{n+1} t_{j}^{n+1}\right| \leq M$ and $\left|\sum_{j=1}^{\max I_{n}} y_{j}\right| \leq M$ and both of these terms have opposite signs. Now for arbitrary $n$ let $k=k_{n}$ be the largest integer such that $\max I_{k} \leq n$. Then $\left|\sum_{j=1}^{n} y_{j}\right|=$ 
$\left|\sum_{j=1}^{\max I_{k}} y_{j}+\sum_{j=\max I_{k}+1}^{n} y_{j}\right| \leq\left|\sum_{j=1}^{\max I_{n}} y_{j}\right|+\left|\sum_{j=\min I_{k+1}}^{n} s_{k+1} t_{j}^{k+1}\right| \leq 2 M$ so $\|y\| \leq 2 M$ as desired.

Note that $b s$ does not have SGHP [consider $t=\{1,-1,1,-1, \ldots\}$ and $I_{k}=\{2 k-1\}$; then $C_{\cup I_{k}} t=\{1,0,1,0, \ldots\} \notin b s$ and likewise the same holds for any subsequence of $\left.\left\{I_{k}\right\}\right]$. Additional spaces with the signed-SGHP can be constructed employing the methods of [BSS].

We next consider the uniform convergence of multiplier convergent series when subsets of the space of multipliers has signed-SGHP. We first establish a lemma.

Lemma 2. Let $\Lambda \subset \lambda$. Let $\sum x_{j}$ be $\Lambda$-multiplier convergent. If the series $\sum_{j=1}^{\infty} t_{j} x_{j}$ do not converge uniformly for $t \in B \subset \Lambda$, then there exist a symmetric neighborhood of $0, V, t^{k} \in B$ and an increasing sequence of intervals $\left\{I_{k}\right\}$ such that $\sum_{j \in I_{k}} t_{j}^{k} x_{j} \notin V$.

Proof : If the series $\sum_{j=1}^{\infty} t_{j} x_{j}$ do not converge uniformly for $t \in B$ , there exist a symmetric neighborhood of $0, U$, such that for every $k$ there exist $t^{k} \in B, m_{k} \geq k$ such that $\sum_{j=m_{k}}^{\infty} t_{j}^{k} x_{j} \notin U$. For $k=1$, let $m_{1}, t^{1} \in B$ satisfy this condition so $\sum_{j=m_{1}}^{\infty} t_{j}^{1} x_{j} \notin U$. Pick a symmetric neighborhood of o, $V$, such that $V+V \subset U$. There exists $n_{1}>m_{1}$ such that $\sum_{j=n_{1}+1}^{\infty} t_{j}^{1} x_{j} \in V$. Then $\sum_{j=m_{1}}^{n_{1}} t_{j}^{1} x_{j}=\sum_{j=m_{1}}^{\infty} t_{j}^{1} x_{j}-\sum_{j=n_{1}+1}^{\infty} t_{j}^{1} x_{j} \notin V$. Put $I_{1}=\left[m_{1}, n_{1}\right]$. Now just continue the construction.

Theorem 3. Let $\Lambda \subset \lambda$ have signed-SGHP and let $\sum x_{j}$ be $\Lambda$-multiplier convergent. Then the series $\sum_{j=1}^{\infty} t_{j} x_{j}$ converge uniformly for $t$ belonging to bounded subsets of $\Lambda$.

Proof: If $B \subset \Lambda$ is bounded and $\sum_{j=1}^{\infty} t_{j} x_{j}$ fails to converge uniformly for $t \in B$, let the notation be as in Lemma 2 . Let $n_{k}, s_{k}$ be as in the definition of signed-SGHP above and $t=\sum_{j=1}^{\infty} s_{j} C_{I_{n_{j}}} t^{n_{j}} \in \Lambda$. Then $\sum t_{j} x_{j}$ does not converge in $X$ since $\sum_{j \in I_{n_{k}}} t_{j} x_{j}=s_{k} \sum_{j \in I_{n_{k}}} t_{j}^{n_{k}} x_{j} \notin V$,i.e., $\sum t_{j} x_{j}$ doesn't satisfy the Cauchy condition.

Remark 4. If $\Lambda=l^{\infty}$, then Theorem 3 implies that any bounded multiplier convergent series is such that the series $\sum_{j=1}^{\infty} t_{j} x_{j}$ converge uniformly for $\|t\|_{\infty} \leq 1$. This statement improves the result for bounded multiplier convergent series given in 8.2.2 of [Sw2], removing the locally convex and sequential completeness assumptions. A (vector) version of Theorem 3 is established in [SS] Lemma 22 under the assumption that the multiplier space has SGHP. A result with the same conclusion as Theorem 3 is given 
in [WLC], Theorem 7, but the assumptions there are quite different, being topological in nature, and difficult to compare.

Remark 5. If $\lambda=m_{0}$ and $\Lambda=M_{0}$,then a series is subseries convergent iff the series is $m_{0}$ or $M_{0}$ multiplier convergent $\left(\operatorname{span} M_{0}=m_{0}\right)$. Then Theorem 3 implies that any subseries convergent series $\sum x_{j}$ is such that the series $\sum_{j=1}^{\infty} C_{\sigma}(j) x_{j}$ converge uniformly for $\sigma \subset \mathbf{N}$ ([Sw2]8.1.2).

Thus, Theorem 3 gives a generalization of the known results for uniform convergence of subseries and bounded multiplier convergent series.

Example 6. Without some type of assumption on the multiplier space $\lambda$, the conclusion of Theorem 3 can fail even when the multiplier space satisfies gliding hump conditions like the weak gliding hump property or the zero gliding hump property ([Sw2]). Let $e^{j}$ be the sequence with a 1 in the $j^{\text {th }}$ coordinate and 0 in the other coordinates. Then $\sum e^{j}$ is $l^{p}-$ multiplier covergent in $\left(l^{p},\|\|_{p}\right)$ for any $1 \leq p<\infty$, but the series $\sum_{j=1}^{\infty} t_{j} e^{j}$ do not converge uniformly for $\|t\|_{p} \leq 1$ [Take $t^{k}=e^{k}, \sum_{j=1}^{\infty} t_{j}^{k} e^{j}=e^{k}$.].

We next consider a multiplier version of the Hahn-Schur Theorem. The classical scalar version of the Hahn-Schur Theorem asserts that if the scalar series $\sum_{j} x_{i j}$ is absolutely convergent for every $i$ and $\lim _{i} \sum_{j \in \sigma} x_{i j}$ exists for every $\sigma \subset \mathbf{N}$ and if $x_{j}=\lim _{i} x_{i j}$, then $\sum x_{j}$ is absolutely convergent and $\sum_{j=1}^{\infty}\left|x_{i j}-x_{j}\right| \rightarrow 0$ or, equivalently, $\lim _{i} \sum_{j \in \sigma}\left(x_{i j}-x_{j}\right)=0$ uniformly for $\sigma \subset \mathbf{N}$ ([Sw2]5.4). By employing the last statement of the conclusion of the classical Hahn-Schur Theorem,versions of the Hahn-Schur Theorem for vector-valued subseries and bounded multiplier convergent series have been given in [Sw2]8.1,8.2, and an abstract version of the theorem which includes certain multiplier convergent series is given in [Sw2]9.3. We now present a version of the Hahn-Schur Theorem for multiplier convergent series when the subset $\Lambda$ of the multiplier space $\lambda$ satisfies the signed-SGHP. We first establish a special case of the theorem.

Lemma 7. Suppose that $\Lambda \subset \lambda$ has signed-SGHP, $\sum_{j} x_{i j}$ is $\Lambda$-multiplier convergent for every $i$ and $\lim _{i} \sum_{j=1}^{\infty} t_{j} x_{i j}=0$ for every $t \in \Lambda$. If $B \subset \Lambda$ is bounded, then $\lim _{i} \sum_{j=1}^{\infty} t_{j} x_{i j}=0$ uniformly for $t \in B$.

Proof : It suffices to show that $\lim _{i} \sum_{j=1}^{\infty} t_{j}^{i} x_{i j}=0$ for any sequence $\left\{t^{i}\right\} \subset B$. Let $U$ be a neighborhood of 0 in $X$ and pick a symmetric neighborhood, $V$, of 0 such that $V+V+V \subset U$. Set $n_{1}=1$ and pick $N_{1}$ such that 
$\sum_{j=N_{1}}^{\infty} t_{j}^{n_{1}} x_{n_{1} j} \in V$. Since $\lim _{i} x_{i j}=0\left(c_{00} \subset \lambda\right)$ for every $j$ and $\left\{t_{j}^{i}: i \in \mathbf{N}\right\}$ is bounded from the $\mathrm{K}$ space assumption on $\lambda, \lim _{i} t_{j}^{i} x_{i j}=0$ for every $j$ ([Sw2]8.2.4) so there exist $n_{2}>n_{1}$ such that $\sum_{j=1}^{N_{1}-1} t_{j}^{i} x_{i j} \in V$ for $i \geq n_{2}$. Pick $N_{2}>N_{1}$ such that $\sum_{j=N_{2}}^{\infty} t^{n_{2}} x_{n_{2} j} \in V$. Continuing this construction produces increasing sequences $\left\{n_{k}\right\},\left\{N_{k}\right\}$ such that $\sum_{l=N_{j}}^{\infty} t_{l}^{n_{j}} x_{n_{j} l} \in V$ and $\sum_{l=1}^{N_{j}-1} t_{l}^{i} x_{i l} \in V$ for $i \geq n_{j}$. Set $I_{j}=\left\{l: N_{j-1} \leq l<N_{j}\right\}$. Define the matrix $M=\left[m_{i j}\right]=\left[\sum_{l \in I_{j}} t_{l}^{n_{j}} x_{n_{i} l}\right]$. We show that $M$ is a signed $K$-matrix in the terminology of [St1], [St2], [Sw2]2.2.4 . First the columns of $M$ go to 0 since $\lim _{i} x_{i l}=0$ for every $l$. Given an increasing sequence $\left\{p_{j}\right\}$, by the signed-SGHP assumption there is a subsequence $\left\{q_{j}\right\}$ of $\left\{p_{j}\right\}$ and signs $\left\{s_{j}\right\}$ such that $t=\left\{t_{j}\right\}=\sum_{j=1}^{\infty} s_{j} C_{I_{q_{j}}} t^{q_{j}} \in \Lambda$. Then $\sum_{j=1}^{\infty} s_{j} m_{i q_{j}}=\sum_{j=1}^{\infty} s_{j} \sum_{l \in I_{q_{j}}} t_{l}^{q_{j}} x_{n_{i} l}=\sum_{j=1}^{\infty} t_{j} x_{n_{i} j} \rightarrow 0$ by hypothesis. Therefore, $M$ is a signed $K$-matrix and by the signed version of the Antosik-Mikusinski Matrix Theorem the diagonal of $M$ goes to 0 ([St1] ,[St2],[Sw2]2.2.4 ). Thus, there exists $N$ such that $m_{i i} \in V$ for $i \geq N$. If $i \geq N$, then $\sum_{l=1}^{\infty} t_{l}^{n_{i}} x_{n_{i} l}=\sum_{l=1}^{N_{i-1}-1} t_{l}^{n_{i}} x_{n_{i} l}+\sum_{l \in I_{i}} t_{l}^{n_{i}} x_{n_{i} l}+\sum_{l=N_{i}}^{\infty} t_{l}^{n_{i}} x_{n_{i} l} \in$ $V+V+V \subset U$ so $\lim _{i} \sum_{l=1}^{\infty} t_{l}^{n_{i}} x_{n_{i} l}=0$. Since the same argument can be applied to any subsequence, it follows that $\lim _{i} \sum_{j=1}^{\infty} t_{j}^{i} x_{i j}=0$

Theorem 8. Suppose that $\Lambda \subset \lambda$ has signed-SGHP, $\sum_{j} x_{i j}$ is $\Lambda$ multiplier convergent for every $i$ and $\lim _{i} \sum_{j=1}^{\infty} t_{j} x_{i j}$ exists for every $t \in \Lambda$. Let $x_{j}=\lim _{i} x_{i j}$ for every $j$. If $B \subset \Lambda$ is bounded, then (i) $\sum x_{j}$ is $\Lambda$-multiplier convergent, (ii) $\lim _{i} \sum_{j=1}^{\infty} t_{j} x_{i j}=\sum_{j=1}^{\infty} t_{j} x_{j}$ uniformly for $t \in B$, (iii) the series $\sum_{j=1}^{\infty} t_{j} x_{i j}$ converge uniformly for $t \in B$.

Proof : Let $t \in \Lambda$. Since the space $\Lambda$ has the signed weak gliding hump property, it follows from Stuart's weak sequential completeness result that $\sum_{j=1}^{\infty} t_{j} x_{j}$ converges and $\lim _{i} \sum_{j=1}^{\infty} t_{j} x_{i j}=\sum_{j=1}^{\infty} t_{j} x_{j}$. ([Sw2] 12.4.1;see also [St1] 3.5); Stuart's result is for the case when $\Lambda$ is a vector space with signed-WGHP, but his proof is valid for a subset with signed-WGHP.

Since $\lim _{i} \sum_{j=1}^{\infty} t_{j}\left(x_{i j}-x_{j}\right)=0$ for every $t \in \Lambda$, Lemma 7 applies and gives (ii).

Suppose that (iii) fails to hold. Then there exists a closed symmetric neighborhood of $0, U$, in $X$ such that for every $i$ there exist $k_{i}>i$, a finite interval $I_{i}$ with $\min I_{i}>i, t^{i} \in B$ such that $\sum_{k \in I_{i}} t_{k}^{i} x_{k_{i} k} \notin U$. Put $i_{1}=1$. By the above, there exist $k_{1}>1, I_{1}$ with $\min I_{1}>i_{1}, t^{1} \in B$ such that $\sum_{k \in I_{1}} t_{k}^{1} x_{k_{1} k} \notin U$. By Theorem 3 there exists $j_{1}$ such that $\sum_{k=j}^{\infty} t_{k} x_{i k} \in U$ for every $t \in B, 1 \leq i \leq k_{1}, j \geq j_{1}$. Set $i_{2}=\max \left\{I_{1}+1, j_{1}\right\}$. Again, 
by the above there exist $k_{2}>i_{2}, I_{2}$ with $\min I_{2}>i_{2}, t^{2} \in B$ such that $\sum_{k \in I_{2}} t_{k}^{2} x_{k_{2} k} \notin U$. Note that $k_{2}>k_{1}$ by the definition of $i_{2}$.

Continuing this construction produces an increasing sequence $\left\{k_{i}\right\}$, an increasing sequence of intervals $\left\{I_{i}\right\}$ and $t^{i} \in B$ such that

(1) $\sum_{k \in I_{i}} t_{k}^{i} x_{k_{i} k} \notin U$.

Define a matrix $M=\left[m_{i j}\right]=\left[\sum_{k \in I_{j}} t_{k}^{i} x_{k_{i} k}\right]$. We claim that $M$ is a signed K-matrix. First, the columns of $M$ converge by hypothesis. Next, given any increasing sequence $\left\{p_{j}\right\}$ there is a subsequence $\left\{q_{j}\right\}$ of $\left\{p_{j}\right\}$ and signs $\left\{s_{j}\right\}$ such that $t=\left\{t_{j}\right\}=\sum_{j=1}^{\infty} s_{j} C_{I_{q_{j}}} t^{q_{j}} \in \Lambda$. Then the sequence $\sum_{j=1}^{\infty} s_{j} m_{i q_{j}}=\sum_{j=1}^{\infty} s_{j} \sum_{l \in I_{q_{j}}} t_{l}^{q_{j}} x_{k_{i} l}=\sum_{j=1}^{\infty} t_{j} x_{k_{i} j}$ converges by hypothesis. Hence, $M$ is a signed K-matrix and the diagonal of $M$ converges to 0 by the signed version of the Antosik-Mikusinski Matrix Theorem ([Sw2]2.2.4). But, this contadicts (1).

A subseries version $\left(M_{0}=\Lambda \subset m_{0}=\lambda\right)$ of the Hahn-Schur Theorem is given in [Sw2]8.1 and a bounded multiplier version of the Hahn-Schur Theorem is given in [Sw2]8.2. Both versions follow from Theorem 3. A (vector) version of Theorem 7 for spaces with SGHP is given in Theorem 25 and Corollary 27 of [SS].

Without some assumption on the multiplier space $\lambda$, the conclusion of Theorem 8 can fail.

Example 9. Let $x_{i j}=e^{j}$ if $1 \leq j \leq i$ and $x_{i j}=0$ if $i<j$. Then $\sum_{j} x_{i j}$ is $l^{p}$-multiplier convergent in $l^{p}$ for $1 \leq p<\infty$ for every $i$. If $t \in l^{p}$, $\sum_{j=1}^{\infty} t_{j} x_{i j} \rightarrow \sum_{j=1}^{\infty} t_{j} e^{j}$ in $l^{p}$. However, the convergence is not uniform for $\|t\|_{p} \leq 1$ [Take $t^{k}=e^{k}$, so $\sum_{j=1}^{\infty} t_{j}^{k} x_{i j}=\sum_{j=1}^{i} t_{j}^{k} e^{j}=e^{k}$ if $i \geq k$.].

There is an abstract version of the Hahn-Schur Theorem which covers certain multiplier convergent series given in [Sw2]9.3; however, the vector version given there uses essentially a strong gliding hump type hypothesis. A (vector) version of Theorem 7 for spaces with SGHP is given in [SS] Theorem 25. Useful vector forms of the signed-SGHP seem to be difficult to formulate. There is a version of Theorem 7 given in [WCC], Theorem 7 , and in $[\mathrm{AP}]$, Theorem 3.1, but the assumptions there are of a topological nature unlike the algebraic signed-SGHP. 


\section{References}

[AP] Aizpuru, A. and Perez-Fernandez, J., Spaces of S-bounded multiplier convergent series, Acta. Math. Hungar., 87, pp. 135-146, (2000).

[B] Boos, J., Classical and modern methods in summability, Oxford University Press, Oxford, (2000).

[BSS] Boos, J.;Stuart, C.;Swartz,C.,Gliding hump properties of matrix domains,Analysis Math., 30, pp. 243-257, (2004).

[D] Day, M., Normed Linear Spaces, Springer-Verlag, Berlin, (1962).

[FP] Florencio, M. and Paul, P., A note on $\lambda$-multiplier convergent series, Casopis Pro Pest. Mat., 113, pp. 421-428, (1988).

[N] Noll, D., Sequential completeness and spaces with the gliding hump property,Manuscripta Math., 66, pp. 237-252, (1960).

[St1] Stuart, C., Weak Sequential Completeness in Sequence Spaces, Ph.D. Dissertation, New Mexico State University, (1993).

[St2] Stuart, C., Weak Sequential Completeness of $\beta$-duals, Rocky Mountain Math. J., 26, pp. 1559-1568, (1996).

[SS] Stuart, C. and Swartz, C., Uniform convergence in the dual of a vectorvalued sequence space,Taiwanese J. Math., 7, pp. 665-676, (2003).

[Sw1] Swartz, C., The Schur lemma for bounded multiplier convergent series, Math. Ann., 263, pp. 283-288, (1983).

[Sw2] Swartz, C., Infinite Matrices and the Gliding Hump, World Sci. Press, Singapore, (1996).

[WCC] [WCC] Wu, J. ; Li, L. ; Cui, C., Spaces of $\lambda$-multiplier convergent series, Rocky Mountain Math. J., 35, pp. 1043-1057, (2005). 


\section{Charles Swartz}

Mathematics Department

New Mexico State University

Las Cruces

NM 88003

USA

e-mail : cswartz@nmsu.edu 